\title{
On Entropy Production in Informational Statistical Thermodynamics
}

\author{
R. Luzzi, A. R. Vasconcellos and J. G. Ramos \\ Instituto de Fisica 'Gleb Wataghin' \\ Universidade Estadual de Campinas, Unicamp \\ Caixa Postal 6165, 13083-970, Campinas, São Paulo, Brasil
}

Received January 13, 1998

\begin{abstract}
We consider the question of the existence of a generalized $\mathcal{H}$-theorem in the context of the variational method in the information-theoretical approach that generates the nonequilibrium statistical operator formalism. After briefly reviewing how the latter provides mechano-statistical foundations for phenomenological irreversible thermodynamics $\Gamma$ the socalled Informational Statistical ThermodynamicsTwe discuss how dissipative phenomena are accounted for by the procedure. Such effects are related to a generalized $\mathcal{H}$-theorem and a weak criterion of positive entropy production. These results are a consequence of the definition of a coarse-grained statistical entropyTresulting from the projection of the full nonequilibrium distribution function on the subspace of the slow relaxing dynamical quantities Tthat are appropriate for the description of the irreversible evolution of the system from the state of initial preparation.
\end{abstract}

\section{Introduction}

Last decades have witnessed extensive and relevant developments in the field of nonlinear nonequilibrium thermodynamics and accompanying kinetic and statistical theories. In large measure this is a result of a growing instrumental capability allowing for detailed measurements in experiments in far-from-equilibrium conditions. Of wide interest is for example the study of electronic devices working under high levels ef excitation and with ultra-short time responses [1] $\Gamma$ and also the case of the physics of open dissipative systems where nonlinearities in conditions sufficiently far away from equilibrium may lead to self-organizing synergetic phenomena [2]. These problems belong to the emerging area of dynamic systems displaying $\Gamma$ the so called $\Gamma$ complex behavior (complex systems for short) encompassing physicalTchemical and biological (as well as others) entities. Phenomenological and statistical thermodynamics play an important role in such situations.

To encompass arbitrary far-from-equilibrium situations $\Gamma$ phenomenological Classical Irreversible Thermodynamics [3] is being superseded by new attempts. Two are Rational Thermodynamics [4] and Extended Irreversible Thermodynamics [5]. On the side of ki- netic and statistical theories several approaches are being pursued $\Gamma$ which have been classified by Zwanzig [6]. Among them the Nonequilibrium Statistical Operators Method (NESOM) may be highlighted $\Gamma$ which is considered to have an appealing structure and seems to be a very effective technique to deal with a large class of experimental situations. This formalism has been formulated by several authors Teither using heuristic arguments [7-10] or projection operator techniques [11-13]. It has been shown [14] that these approaches can be brought together under a unifying variational method $\Gamma$ which places the formalism of the NESOM within the scope of Jaynes' Predictive Statistical Physics [15]. A classical approachTas the one to be used in this paper $\Gamma$ and the foundations of a generalized Boltzmann-like approach is described in reference [16]. The formalism is referred to as the MaxEnt-NESOM.

The MaxEnt-NESOM appears as an appropriate formalism to yield mechanico-statistical foundations to phenomenological irreversible thermodynamics. It provides for the construction of the Informational Statistical Thermodynamics (also referred to as Informationtheoretic Thermodynamics) $\Gamma$ originating in the work of HobsonT Nettleton $\Gamma$ and others [17] (a brief review and details of application of the MaxEnt-NESOM in 
statistical thermodynamics is given in reference [18]). This fact brings to the fore a fundamental question in nonequilibrium thermodynamics 5 namely which is the origin of irreversibility (or the origin of the so called time arrow problem [19]) and the definition of an entropy and entropy production functions Tand the sign of the latter. This is a quite difficult Thowever engagingI problem that has associated a considerable amount of controversy [see for example TLetters Section in Physics Today 47 (11)Гpp. 11-15 and 115-117ГNovember 1994]. The question has been subsumed by Leon Rosenfeld as "to express in a precise formalism [the] complementarity between the thermodynamic or macroscopic aspect and the atomic one" [20]. Several approaches $\Gamma$ seemingly different at first sight $\Gamma$ have been produced $\Gamma$ beginning with the genial contributions of Ludwig Boltzmann [21]. Some Schools set irreversibility at the level of probability distributions but together with methods of Teither discarding microscopic information that is unnecessary for predicting the behavior of the macroscopic state of the system (on the basis of information theory) Tor introducing a dynamic of correlations; they are compared in reference [22]. More recently some approaches have relied on a description in terms of a dynamic origin of irreversibility as associated to ergodic properties of chaotic-like systems [23 I24]. In Mackey's line of thought irreversibility appears hidden behind the concept of irreversibility in a rather abstract mathematical wayTand no connection is made with the concept of entropy production. On the other hand Hasegawa and Driebe's work deals with irreversibility for a particular class of chaotic systems; how it can be extended to quite general thermodynamic situations is an open matter. As pointed out by J.L. Lebowitz [25] all these approaches contain interesting and useful ideas and can be illuminating when properly applied.

We here deal with the question strictly within the framework of Informational Statistical Thermodynamics. Therefore $\mathrm{C}$ it must be understood that the functions that in what follows are referred to as entropy and entropy production are those that the theory defines. J. MeixnerTover twenty years ago in paper that did not obtain a deserved diffusion [26] Tgave some convincing arguments to show that it is very unlikely that a nonequilibrium state function playing the role of the entropy may be uniquely defined. Summarizing his ideas one may assert that the conclusion reached by him is that such a functionTeither cannot be defined $\Gamma$ or it may be done so in an infinite number of ways. A softened form of this idea was advanced by Grad over thirty year ago [27]. Exploring recent literature on this question these conjectures seem to hold true in a more restricted sense [28]. It ought to be noticed that the MaxEnt-NESOMT as indicated $\Gamma$ provides for the construction of Informational Statistical Thermodynamics and then gives microscopic foundations to phenomenological thermodynamic theories $\Gamma$ in particular it covers and generalizes Extended Irreversible Thermodynamics [18]. MoreoverTMaxEnt-NESOM provides a nonlinear quantum transport theory of large scope [29] That implies in a far-reaching generalization of Mori's theory [30] Ttogether with an accompanying response function theory for far-from-equilibrium systems [14] Tand a generalized Boltzmann transport theory [16]. MaxEntNESOM has been applied to the study of ultrafast relaxation processes in the so-called photoinjected plasma in semiconductors with particular success $\Gamma$ in the sense of obtaining very good agreement between theory and experiment [31]. Such kind of experiments can be used to satisfactorily allow for the characterization and measurement of nonequilibrium thermodynamic variables like nonequilibrium temperature $\Gamma$ chemical potentials $\Gamma$ etc. Twhich are concepts derived from the entropy function that the theory defines in a similar way to equilibrium and local equilibrium theories [32]. Since they depend on such entropy-like function they are usually referred to as quasi-temperature Tquasi-chemical potentialsTetc. MoreoverTwithout attempting a rigorous approach $\Gamma$ we simply call the attention to the fact that the formalism here presented appears as providing a kind of partial unification of several - apparently differentiated - approaches to the subject: First $\Gamma$ the formalism begins with a derivation within the framework of Jaynes-style informational approach $\Gamma$ and $\Gamma$ therefore $\Gamma$ the (informational) entropy that the method introduces is dependent on a restricted set of variables. This is a result that this entropy is a projection on the space of such contracted set of variables of the fine-grained Gibbs entropy (as later on described and depicted in Fig. 1. The latter is obtained $\Gamma$ as shown in next sectionTon the basis of a memory-dependent MaxEnt construction. SecondT the connection with the approach of the Brussels-Austin School (subdynamics of correlations) partially appears with the introduction in this particular MaxEnt approach of an ad hoc hypothesis that introduces irreversibility from the outset $\Gamma$ consisting in a mimic of Prigogine's dynamical condition for dissipativity [33]. Additional discussions on the equiv- 
alence of both approaches have been provided by several authors [34]. Finally it can be shown that the method contains Boltzmann's equation and $\mathcal{H}$-theorem as particular cases [16]. It is also worth noticing that the MaxEnt-NESOM provides microscopic foundations for an extension of Prigogine's generalized Nonequilibrium ThermodynamicsTimplying in generalizations to arbitrary nonequilibrium conditions (that is $\Gamma$ outside the local equilibriumn approximation) of Prigogine's theorem of minimum entropy production in the linear regime near equilibrium $\Gamma$ Glansdorff-Prigogine's thermodynamic criterion for evolutionTand (in)stability criterion $\Gamma$ which are - within the theory - all of them $\Gamma$ a consequence of the convexity of the MaxEnt entropy [35].

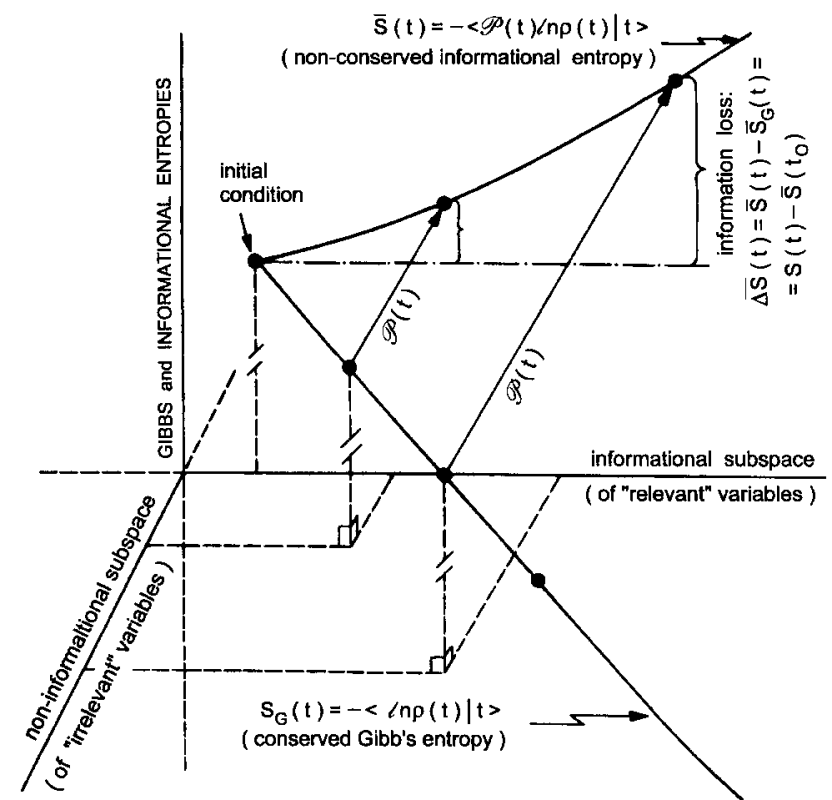

Figure 1. Graphical description of the macroscopic state of the system in terms of the MaxEnt-nonequilibrium statistical distribution. The projection of $\ln \rho$ - depending on the instantaneous state of the system [see Eq. (14)] - introduces a coarse-graining procedure consisting in projecting onto the subspace of the "relevant" variables associated to the informational constraints in MaxEnt.

Therefore $\Gamma$ it appears to be pertinent to try to discuss how these questions of irreversibility and $\mathcal{H}$ theorems $\Gamma$ as well as entropy and entropy production $\Gamma$ manifest themselves and behave in the framework of Informational Statistical Thermodynamics. This is done in the next sections that are organized in the following way: in section II we present a short description of the MaxEnt-NESOM in a classical approach $\Gamma$ whereas in section IIITafter a brief description of the MaxEntNESOM approach to irreversible thermodynamicsTwe discuss the question of the definitions of the MaxEnt entropy and entropy production the question of irreversibility and an accompanying MaxEnt generalized $\mathcal{H}$-theorem $\Gamma$ as well as a weak principle of positive entropy production. Last section summarize the results and comment upon them.

\section{Brief summary of the theoretical background}

The first $\Gamma$ and fundamental $\Gamma$ step in MaxEntNESOM is the choice of the basic set of variables that are appropriate for the characterization of the macroscopic state of the system. This involves a contracted description in terms of $\mathrm{T}$ say $\mathrm{T}$ the dynamical quantities $P_{1}(\Gamma), P_{2}(\Gamma), \ldots, P_{n}(\Gamma) \Gamma$ where $\Gamma$ is a point in phase space characterizing the state of the system at the microscopic classical-mechanical level. The MaxEntNESOM statistical distribution is a functional of these quantities $\Gamma$ to be called $\rho\left(\left\{P_{j}(\Gamma)\right\} \mid t\right)$, or $\Gamma$ for short $\Gamma$ $\rho(\Gamma \mid t)$. The macroscopic (or mesoscopic) state is characterized by a point in Gibbs space $\Gamma$ composed $\Gamma$ at time $t \Gamma$ of the set of macrovariables $Q_{1}(t), \ldots, Q_{n}(t)$, which are the average values of the $P_{j}$ Ti.e. $Q_{j}(t)=$ $\operatorname{Tr}\left\{P_{j} \rho(\Gamma ; t)\right\}$. The choice of the basic variables is assisted by Bogoliubov's procedure of contraction of description based on a hierarchy of relaxation times [36] $\Gamma$ and - to it related - the ideas set forwardTamong othersTby Mori [30 137] CZubarev [10] Tand Peletminskii [9]. They consist in introducing a separation of the total Hamiltonian into two partsTnamely

$$
H=H_{0}+H^{\prime},
$$

In this Eq. (1) $H_{0}$ is a "relevant" (or secular) part composed of the kinetic energies of the free subsystems and some of the interactions Tnamely those strong enough to have associated very short relaxation times (meaning those much smaller than the characteristic time scale of the experiment) $\Gamma$ and possessing certain symmetry properties. The other term $\Gamma H^{\prime} \Gamma$ contains the interactions related to long-time relxation mechanisms. The symmetry characteristics of the strong interactions depend on the problem under consideration: The required symmetry - to be called Zubarev-Peletminskii symmetry condition - is that 


$$
\left\{P_{j}, H_{0}\right\}=\sum_{K} \alpha_{j k} P_{k},
$$

where the left hand side is Poisson's bracket $\Gamma$ and $\alpha_{j k} \Gamma$ are $c$-numbers determined by $H_{0}$. It should be noticed that quantities $P_{j}$ can be dependent on the space variable (or even $\Gamma$ in the classical description $\Gamma$ dependent on momentum) and then the coefficients $\alpha$ can also be differential operators. Eq. (2) provides a closure condition for the choice of the basic set of variables in a step by step procedure described elsewhere:[14П6П18].

Assuming that the basic set $\left\{P_{j}(\Gamma)\right\}$ has been cho- sen the nonequilibrium statistical distribution (NSD) is constructed $\Gamma$ within the context of Jaynes' Predictive Statistical Mechanics [15] Tusing the principle of maximization of "statistical-informational entropy MaxEnt for short Twith memory and ad hoc hypotheses [14П6]. Summarizing the results that are of relevance in next sections $\Gamma$ the variational procedure produces a statistical distribution - containing nonlocal in space and memory effectsTas well as irreversibility - that is a functional of an instantaneous in time auxiliary (or coarsegrained) Gibbsian-like distribution given by

$$
\bar{\rho}(\Gamma \mid t, 0)=\exp \left\{-\phi(t)-\sum_{j=1}^{n} \int d^{3} r F_{j}(\vec{r}, t) P_{j}(\Gamma \mid \vec{r})\right\},
$$

where $\phi(t)$ ensures its normalization $\Gamma a n d F_{j}(\vec{r}, t)$ are the Lagrange multipliers that the method introduces. In the derivation an important coarse-grained conditon is imposed (of fundamental relevance in what follows) namely that

$$
Q_{j}(t)=\int d \Gamma \rho_{w}(\Gamma \mid t) P_{j}(\vec{r})=\int d \Gamma \bar{\rho}(\Gamma \mid t) P_{j}(\vec{r}),
$$

FurthermoreTthe fine-grained NSD is given in terms of the coarse-grained one in the form

$$
\ln \rho_{w}(\Gamma \mid t)=\ln \bar{\rho}(\Gamma \mid t, 0)-\int_{t_{0}}^{t} d t^{\prime} \mathcal{W}\left(t, t^{\prime}\right) \frac{d}{d t^{\prime}} \ln \bar{\rho}\left(\Gamma \mid t^{\prime}, t^{\prime}-t\right),
$$

where $\mathcal{W}$ is an appropriate weight function defined in [14П16] $\Gamma$ and the first time $\Gamma t^{\prime} \Gamma$ in the argument of $\bar{\rho}$ refers to the time dependence of the Lagrange multipliers and the second $\Gamma t^{\prime}-t$, to the time dependence of quantities $P_{j}$ which evolve according to Hamilton's equations of motion. Moreover $\Gamma t_{0}$ is the initial time $\Gamma$ that is the one of preparation of the system and $t$ the time when a measurement is performed. Two important consequences follow from Eq.(5): one is that the initial condition has been fixed $\Gamma$ and it is expressed by

$$
\rho_{w}\left(\Gamma \mid t_{0}\right)=\bar{\rho}\left(\Gamma \mid t_{0}, 0\right)
$$

and the other that the NSD can be written as the sum of two terms as

$$
\rho_{w}(\Gamma \mid t)=\bar{\rho}(\Gamma \mid t, 0)+\rho_{w}^{\prime}(\Gamma \mid t)
$$

where $\bar{\rho}$ is given in Eq. (3) and

$$
\rho_{w}^{\prime}(\Gamma \mid t)=\sum_{k=1}^{\infty} \frac{1}{k !}\left[\int_{t_{0}}^{t} d t^{\prime} \mathcal{W}\left(t, t^{\prime}\right) \hat{\sigma}\left(\Gamma \mid t^{\prime}, t^{\prime}-t\right)\right]^{k} \bar{\rho}(\Gamma \mid t, 0),
$$

with

$$
\hat{\sigma}\left(\Gamma \mid t^{\prime}, t^{\prime}-t\right)=-\frac{d}{d t^{\prime}} \ln \bar{\rho}\left(\Gamma \mid t^{\prime}, t^{\prime}-t\right)
$$


Eq. (7) indicates that the NSD is composed of an instantaneous ("frozen" or coarse-grained) generalized Gibbsian distribution $\bar{\rho}$ Twhich also defines the instantaneous values of the averages of the basic variablesTCf. Eq. (4) $\Gamma$ plus a deviation that accounts for the microscopic processes that produce dissipative effects in the system Tto be better evidenced later on in this paper. The particular choice of the form of the Lagrange multipliers that the method introduces and the properties of the accompanying weight function $\mathcal{W}$ Tare responsible $\Gamma$ first $\Gamma$ for leading to Eq. (6) $\Gamma$ which indicates that correlations affecting the basic variables prior to time $t_{0}$ are ignored $\Gamma$ and $\Gamma$ second $\Gamma$ for the irreversible evolution of the macroscopic state of the system for $t>t_{0}[14 \Pi 6]$. It may be said that in this way it has been introduced a particular Stosszahlansatz.

Moreover Tit can be shown that there exists a timedependent projection operator $\mathcal{P}(t)$ defined as

$$
\mathcal{P}_{w}(t) A(\Gamma)=\sum_{i, j=0}^{n} P_{i}(\Gamma) \tilde{C}_{i j}^{-1}(t)\left\{P_{j}(\Gamma) ; A(\Gamma) \mid t\right\},
$$

where index zero refers to a $P_{0}(\Gamma)$ taken as the unit operatorTand $F_{0}(t)$ is $\phi(t)$, and

$$
\tilde{C}_{i j}(t)=\left\{P_{i}(\Gamma) ; P_{j}(\Gamma) \mid t\right\},
$$

has been introduced $\Gamma$ which is a supercorrelation function $\Gamma$ that for any pair of dynamical quantities $A$ and $B$ is given by

$$
\{A(\Gamma) ; B(\Gamma) \mid t\}=\int d \Gamma A(\Gamma) Y_{w}(\Gamma 0 B(\Gamma) \bar{\rho}(\Gamma \mid t, 0)
$$

with

$$
Y_{u}(\Gamma)=1+\sum_{k=1}^{\infty} \frac{1}{k !}\left[\int_{t_{0}}^{t} d t^{\prime} \mathcal{W}\left(t, t^{\prime}\right) \sigma\left(\Gamma \mid t^{\prime}, t^{\prime}-t\right)\right]^{k} .
$$

For simplicity we have omitted the possible dependence of quantities $P_{j}$ on the space variable.

This time-dependent projection operator has the property that $\Gamma$ used in conjunction with the coarse-graining definition of Eq. (4) Tprojects at any time the logarithm of the NSD over the logarithm of the auxiliary (coarsegrained) distributionTnamely

$$
\mathcal{P}_{w}(t) \ln \rho_{w}(\Gamma \mid t)=\ln \bar{\rho}(\Gamma \mid t, 0) ;
$$

we shall see the relevance of this result as we proceed in next sections.

Finaly the MaxEnt-NESOM provides the framework for the derivation of the equations of evolution for the basic variablesTa nonlinear generalized transport theory. Differentiation in time of Eq. (4) leads us to the evolution equation

$$
\frac{\partial}{\partial t} Q_{j}(\vec{r}, t)=\int d \Gamma\left\{P_{j}(\Gamma, \vec{r}), H(\Gamma)\right\} \rho_{w}(\Gamma \mid t),
$$

which using Eqs. (7) $Г(1) \Gamma(2)$ and (4) we can rewrite as

$$
\frac{\partial}{\partial t} Q_{j}(\vec{r}, t)=J_{j}^{(0)}(\vec{r}, t)+J_{j}^{(1)}(\vec{r}, t)+\mathcal{J}_{j}(\vec{r}, t),
$$

where

$$
\begin{aligned}
J_{j}^{(0)}(\vec{r}, t) & =\int d \Gamma\left\{P_{j}(\Gamma, \vec{r}), H_{0}(\Gamma)\right\} \bar{\rho}(\Gamma \mid t, 0), \\
J_{j}^{(1)}(\vec{r}, t) & =\int d \Gamma\left\{P_{j}(\Gamma, \vec{r}), H^{\prime}(\Gamma) \bar{\rho}(\Gamma \mid t, 0),\right.
\end{aligned}
$$




$$
\mathcal{J}_{j}(\vec{r}, t)=\int d \Gamma\left\{P_{j}(\Gamma, \vec{r}), H^{\prime}(\Gamma)\right\} \bar{\rho}_{w}^{\prime}(\Gamma \mid t) .
$$

As we shall see only the collision integral $\mathcal{J}$ is associated to dissipative processes $\Gamma$ and we notice that it can be alternatively written in the interesting form

$$
\mathcal{J}_{j}(\vec{r}, t)=\int_{t_{0}}^{t} W\left(t, t^{\prime}\right)\left\{\left\{P_{j}(\Gamma, \vec{r}), H^{\prime}(\Gamma)\right\} ; \hat{\sigma}\left(\Gamma \mid t^{\prime}, t^{\prime}-t\right) \mid t\right\},
$$

where $\sigma$ is given by Eq. (9) $\Gamma$ and $\mathcal{J}$ involves then the super-correlation function [defined in Eq. (12)] of the time evolution of quantity $P_{j}$ with the part of the Hamiltonian associated to the slow relaxing processes and the quantity $\hat{\sigma}$ associated $\Gamma$ as shown in next section $\Gamma$ to the MaxEnt-NESOM entropy production $\Gamma$ and also depending on $H^{\prime}$.

Evidently the collision integral of Eq. (18) is extremely difficult to handle; howeverTit can be brought into a form that allows for a more accessible and pratical mathematical handling Tnamely [29]

$$
\mathcal{J}_{j}(\vec{r}, t)=\sum_{n=2}^{\infty} J_{j}^{(n)}(\vec{r}, t),
$$

where the $J_{j}^{(n)}$ are partial collision integrals that are instantaneous in time and organized according to increasing powers $n$ of the strengths of the interactions contained in $H^{\prime}$. The lowest order $\Gamma n=2$ only $\mathrm{T}$ is an instantaneous in time approximation referred to as the quasi-linear theory of relaxation [38]. These partial collision operators $\Gamma$ which have an important role in the definition of the MaxEnt-NESOM entropy production function $\Gamma$ are composed of several terms consisting of (1) the mechanical effects of collisions (in order $n$ ) averaged over the auxiliary coarse-grained ensemble $\Gamma(2)$ terms that account for the evolution of the thermodynamic state of the system $\Gamma$ and (3) $\Gamma$ for $n>2 \Gamma$ terms arising from memory effects [29]. They are of ever larger complexity with increasing $n \Gamma$ but a truncationprocedure may be introduced in the series of Eq. (19) up to a certain order $n$ in the interaction. It may be noticed that the MaxEnt-NESOM provides a relevant and quite practical formalism lleading to theoretical results in very good agreement with experiment $\Gamma$ when dealing with relaxation processes in ultrafast laser spectroscopy in highly photoexcited semiconductors [31].

Having thus briefly reviewed the fundamentals of the method we proceed in next section to the discussion of the associated thermodynamics.

\section{MaxEnt-Entropy, entropy production, and generalized $\mathcal{H}$-theorem}

Entropy has a very special status in Physics $\Gamma$ a concept well established in equilibrium but elusive in nonequilibrium conditions when it requires an extension in a way applicable to open systems and to situations far from equilibrium. Moreover $\Gamma$ of course $\Gamma$ this must be done in such a way that one recovers the results of equilibrium thermodynamics and classical linear irreversible thermodynamics. In order to introduce a definition that may play the role of an entropy-like function for the most general situations $\Gamma$ it must be faced $\Gamma$ first of all $\Gamma$ the fundamental problem of the choice of the $\Gamma$ in some sense $\Gamma$ complete basic set of macrovariables. In both $\Gamma$ phenomenological irreversible thermodynamics and MaxEnt-NESOM - as discussed in the previous section - there is not a wholly satisfactory way to perform such choice $\Gamma$ and then $\Gamma$ we stressed that one is restricted to the use of a truncated set deemed appropriate for each case under consideration. Of course in each case it is required to perform a careful analysis of the validity of the truncation $\Gamma$ that is $\Gamma$ an evaluation resorting to some kind of criterion - of the information lost in the process showing that it is in fact smaller than the one mantained (for a particular example see the third of references [18]).

Keeping this point in mind 5 the MaxEnt-NESOM allows for the definition of what we call MaxEnt entropy 
function which can be identified with those of phenomenological irreversible thermodynamics [5] Tnamely

$$
\bar{S}(t)=-\int d \Gamma \rho_{w}(\Gamma \mid t) \ln \bar{\rho}(\Gamma \mid t, 0)
$$

where we have taken Boltzmann constant as unit. Using the definition of $\bar{\rho}$ given by Eq. (3) we can also write that $\Gamma$

$$
\begin{aligned}
\bar{S}(t) & =\phi(t)+\sum_{j=1}^{N} \int d^{3} r F_{j}(\vec{r}, t) \int d \Gamma \rho_{w}(\Gamma \mid t) P_{j}(\Gamma \mid \vec{r})= \\
& =\phi(t)+\sum_{j=1}^{n} \int d^{3} r F_{j}(\vec{r}, t) Q_{j}(\vec{r}, t),
\end{aligned}
$$

which is a function of the state variables $Q_{j}$ only since

$$
d \bar{S}(t)=\sum_{j=1}^{n} \int d^{3} r F_{j}(\vec{r}, t) d Q_{j}(\vec{r}, t),
$$

a Pfaffian formTconsisting in a generalized Gibbs relation Twhere

$$
F_{j}(\vec{r}, t)=\delta \bar{S}(t) / \delta Q_{j}(\vec{r}, t),
$$

that isT the MaxEnt-Lagrange multipliers are thus the functional derivatives of the MaxEnt entropy. In deriving Eq. (22) use was made of the relation

$$
d \phi(t)=\sum_{j=1}^{n} \int d^{3} r Q_{j}(\vec{r}, t) d F_{j}(\vec{r}, t)
$$

where

$$
Q_{j}(\vec{r}, t)=-\delta \phi(t) / \delta F_{j}(\vec{r}, t)
$$

and $\delta$ stands for functional differentiation.

Eqs. (23) and (25) tell us that variables $Q_{j}$ and $F_{j}$ are thermodynamically conjugated to each other (these expressions are the generalization of the corresponding relations in equilibrium). This is a consequence of the conditions imposed on the weight function $\mathcal{W}\left(t, t^{\prime}\right)$ and the coarse-graining property of Eq.(4). Eqs. (23)T or (25) Tcan be considered as nonequilibrium equations of state $\Gamma$ and Eq. (21) is a Legendre-like transformation that defines $\phi(t)$ as a Massieu-Planck functional derived from the MaxEnt entropy.

The MaxEnt entropy production function is then $\Gamma$ on account of Eq. (22) Tgiven by

$$
\sigma(t)=\frac{d}{d t} \bar{S}(t)=\sum_{j=1}^{n} \int d^{3} r F_{j}(\vec{r}, t) \frac{\partial}{\partial t} Q_{j}(\vec{r}, t),
$$

where the time variation of the quantities $Q_{j}$ is governed by Eq.(16)Tand then composed of three terms associated to $J^{(0)} \Gamma J^{(1)}$ and and $\mathcal{J}$. However $\Gamma$ as shown in Appendix ITit follows that

$$
\sigma(t)=\sum_{j=1}^{n} \int d^{3} r F_{j}(\vec{r}, t) \mathcal{J}_{j}(\vec{r}, t)
$$

which implies that only the collision integral $\mathcal{J}$ contributes to the MaxEnt-entropy production.

Moreover $\Gamma$ the MaxEnt-entropy production admits alternative forms as shown in Appendix IIT the quantity $\hat{\sigma}\left(\Gamma \mid t_{1}, t_{2}\right) \Gamma$ defined by Eq. (9) Thas the property that its average value is the NESOM-entropy production function $\Gamma$ namely 


$$
\begin{aligned}
\sigma(t) & =\int d \Gamma \hat{\sigma}(\Gamma \mid t, 0) \rho_{w}(t)= \\
& =\int d \Gamma \hat{\sigma}(\Gamma \mid t, 0)\left[\bar{\rho}(t, 0)+\rho_{w}^{\prime}(t)\right]= \\
& =\int d \Gamma \hat{\sigma}(\Gamma \mid t, 0) \rho_{w}^{\prime}(t)=\sum_{j} \int d^{3} r F_{j}(\vec{r}, t) \frac{\partial}{\partial t} Q_{j}(\vec{r}, t),
\end{aligned}
$$

where we have used Eqs. (2) and (4).

ClearlyГon account of Eq. (28) $\rho_{w}^{\prime}$ of Eq. (8) contains the information on the macroscopic state of the system that contributes to $\sigma(t)$; hence $\mathrm{T}$ the auxiliary (coarse-grained) ensemble characterized by $\bar{\rho}$ is relaxation free. We can also state that all relaxation pro-

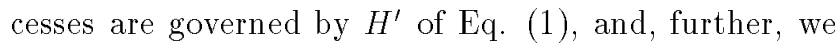
have that

$$
\bar{S}(t)=-\int d \Gamma \rho_{w}(\Gamma \mid t) \mathcal{P}(t) \ln \rho_{w}(\Gamma \mid t),
$$

and then

$$
\sigma(t)=-\int d \Gamma \rho_{w}(\Gamma \mid t) \frac{d}{d t}\left[\mathcal{P}(t) \ln \rho_{w}(\Gamma \mid t)\right],
$$

where $\mathcal{P}(t)$ is the time-dependent projection operator of Eq. (10). Here we have used Eq. (14) Trelating the definition of the MaxEnt entropy and the existence of a non- vanishing MaxEnt- entropy production to the coarse-graining operation performed by $\mathcal{P}(t) \Gamma$ projecting over the sub-space of basic quantities $P_{j}$ which are quasi-conserved under $H_{0}$ [Cf. Eq. (2)].

The MaxEnt-entropy production can be related to those of phenomenological thermodynamicsTand from it to derive generalizations of Prigogine's theorem of minimum entropy production in the linear regime Tand in the nonlinear one of Glansdorff-Prigogine's evolution criterion $\Gamma$ and (in)stability criterion [35]. A connection with phenomenological irreversible thermodynamics has been established $\Gamma$ and $\Gamma$ furthermore $\Gamma$ the foundations for the inclusion of fluxes and higher order fluxes of quasi-conserved variables $\Gamma$ as done in extended irreversible thermodynamicsTarise naturally in the MaxEnt-NESOM based Informational Statistical Thermodynamics. FurthermoreTone can obtain equations of evolution of the so-called Maxwell-CattaneoVernotte type which replace the constitutive equations of classical linear irreversible thermodynamicsTthus removing conceptual and practical difficulties of the latter [18].

One point that is presently missing in the attempt to extract from this Informational Statistical Thermodynamics (and thus to also provide for a reasonable criterion in phenomenological irreversible thermodynamics) is the sign of the entropy production function. This is defined as non negative in extended irreversible thermodynamicsTbut such property does not follows immediatly from $\sigma$ of Eq. (27). Operator $\mathcal{J}$ on which depends has an extremely complicated expression $\Gamma$ even in its alternative form given by Eq. (19). There is only one manageable case $\Gamma$ the quasi-linear theory of relaxation $\Gamma$ when $\sigma$ is definite positive $\Gamma$ as shown in [39].

However $\Gamma$ we can show that in this informational nonequilibrium statistical thermodynamics there follows a generalized $\mathcal{H}$-theorem, in the sense of Jancel [40]Г and a weak principle of entropy production. For that purpose we take into account that the definition of the MaxEnt entropy allows us to write

$$
\bar{S}(t)-\bar{S}\left(t_{0}\right)=-\int d \Gamma\left[\rho_{w}(\Gamma \mid t) \ln \bar{\rho}(\Gamma \mid t, 0)-\rho_{w}\left(\Gamma \mid t_{0}\right) \ln \bar{\rho}\left(\Gamma \mid t_{0}, 0\right] .\right.
$$

But $\Gamma$ because of the initial condition of Eq. (6) we have that $\ln \rho_{\varepsilon}\left(\Gamma \mid t_{0}, 0\right)=\ln \rho_{w}\left(\Gamma \mid t_{0}\right)$ and $\Gamma$ further $\Gamma$ Gibbs entropyГnamely 


$$
S_{G}(t)=-\int d \Gamma \rho_{w}(\Gamma \mid t) \ln \rho_{w}(\Gamma \mid t)
$$

is conserved $\Gamma$ that is $\Gamma$ it is constant in time. Then it follows that

$$
\begin{aligned}
\operatorname{bar} S(t)-\bar{S}\left(t_{0}\right) & =-\int d \Gamma \rho_{w}(\Gamma \mid t, 0)\left[\ln \bar{\rho}(\Gamma \mid t, 0)-\ln \rho_{w}(\Gamma \mid t)\right]= \\
& \left.=-\int d \Gamma \rho_{w}(\Gamma \mid t)\left[\mathcal{P}_{w}(t)-1\right] \rho_{w}(\Gamma \mid t)\right]
\end{aligned}
$$

where $\mathcal{P}$ is the projection operator of Eqs. (10) and (14). Hence $\Gamma$

$$
\bar{S}(t)-\bar{S}\left(t_{0}\right)=\bar{S}(t)-S_{G}(t),
$$

with $S_{G}$ being Gibbs' (fine-grained) statistical entropy of Eq.(32).

Recalling that the coarse-graining condition of Eq. (4) ensuresTbesides the definition of the Lagrange multipliers $F_{j}$ which according to Eq. (23) are differential coefficients of the entropyГthe simultaneous normalization of $\rho_{w}$ and $\bar{\rho}$ Гwe can write Eq. (31) in the form

$$
\begin{aligned}
\Delta \bar{S}(t) & =\bar{S}(t)-\bar{S}\left(t_{0}\right)=\bar{S}(t)-S_{G}(t)= \\
& =-\int d \Gamma\left[\rho_{w}(\Gamma \mid t) \ln \bar{\rho}_{w}(\Gamma \mid t, 0)-\rho_{w}(\Gamma \mid t) \ln \rho_{w}(\Gamma \mid t)+\rho_{w}(\Gamma \mid t)-\bar{\rho}(\Gamma \mid t, 0)\right] .
\end{aligned}
$$

This quantity $\Delta \bar{S}$ cancels for $\rho_{w}=\bar{\rho}$ (i.e. for null $\rho_{w}^{\prime}$ of Eq. (7)) Tand its variation is

$$
\begin{aligned}
\delta \Delta \bar{S}(t) & =\int d \Gamma \delta \rho_{w}(\Gamma \mid t) \ln \left[\rho_{w}(\Gamma \mid t) / \bar{\rho}(\Gamma \mid t, 0)\right]= \\
& =\int d \Gamma \delta \rho_{w}(\Gamma \mid t) \ln \left[1+\rho_{w}^{\prime}(\Gamma \mid t) / \bar{\rho}_{w}(\Gamma \mid t, 0)\right],
\end{aligned}
$$

where we used the separation of $\rho_{w}$ given by Eq. (7).

The variation in Eq. (36) vanishes for $\rho_{w}=\bar{\rho}$ Tand $\Gamma$ as shownTalso vanishes $\Delta \bar{S}$ Tso it follows that $\Delta \bar{S}(t)$ is a minimum for $\rho_{w}=\bar{\rho}$, when it is zero $\Gamma$ and positive otherwiseTnamely

$$
\Delta \bar{S}(t) \geq 0
$$

which defines for MaxEnt-NESOM the equivalent of Jancel's generalized $\mathcal{H}$-theorem [40]. It should be noticed that the inequality of Eq. (37) can be interpreted as the fact that $\Gamma$ as the system evolves in time from the initial condition of preparation under the governing action of the nonlinear generalized transport equations (16) The MaxEnt entropy cannot decrease $\Gamma$ orTbecause of Eq. (37) Tthe MaxEnt entropy is always larger than
Gibbs statistical entropy. These results reproduce for the MaxEnt-NESOM described in section IITthose obtained by del Rio and Garcia-Colin [41].

Using the definition for the MaxEnt-NESOM entropy production function we can rewrite Eq. (37) as

$$
\int_{t_{0}}^{t} d t^{\prime} \int d^{3} r \sigma\left(\vec{r}, t^{\prime}\right) \geq 0
$$

where we have introduced the MaxEnt-entropy production function $\Gamma$ dependent on the space and time variables $\Gamma$

$$
\sigma(\vec{r}, t)=\sum_{j=1}^{n} F_{j}(\vec{r}, t) \frac{\partial}{\partial t} Q_{j}(\vec{r}, t)=
$$




$$
=\sum_{j=1}^{n} F_{j}(\vec{r}, t) \mathcal{J}_{j}(\vec{r}, t) .
$$

Eq. (38) does not prove that $\sigma(t)$ is a monotonically increasing function of timeTas required by phenomenological irreversible thermodynamic theories. We have only proved the weak condition that as the system evolves $\sigma(t)$ is predominantly definite positive. We also insist on the fact that this result is a consequence of the presence of the contribution $\rho_{w}^{\prime}$ to $\rho_{w}$ Twhich is then - as stated previously - the part that accounts for $\Gamma$ in the description of the macroscopic state of the system The processes that generate dissipation. Furthermore The MaxEnt entropy with the evolution property of Eq. (37) is the coarse-grained entropy of Eq. (29) I the coarse-graining being performed by the action of the projection operator $\Gamma$ which extracts the part of the logarithm of Gibbs entropy projected onto the subspace spanned by the dynamical quantities associated to the constraints [Cf. Eq. (4)] imposed on the system. This is graphically illustrated in Fig. 1. Clearly then the MaxEnt entropy thus defined depends on the choice of the basic set of macroscopic variables $\Gamma$ whose completeness in a purely thermodynamic sense cannot be indubitably asserted. We restate that in each particular problem under consideration the information lost in the particular truncation on the set of basic variables must be carefully evaluated [18;42]. Two other properties of the MaxEnt entropy function is that it is a maximum compatible with the constraints of Eq. (4) when they are given at the specific time $t \Gamma$ or that $\bar{\rho}$ maximizes $\bar{S}(t)$ when subjected to normalization and such constraints $\Gamma$ and second one recovers the proper values in equilibrium. This particular propertyГ which tells us that $\bar{S}(t)$ is a convex function in the space of thermodynamic states $\Gamma$ is the one that ensures that in the framework of Informational Statistical Thermodynamics are contained Prigogine's theorem of minimum entropy production in the linear regime around equilibrium Tand Glansdorff-Prigogine's thermodynamic principles of evolution and nonlinear conditions [35].

\section{Concluding remarks}

As remarked in the Introduction $\Gamma$ phenomenologi- cal nonlinear nonequilibrium thermodynamics and its statistical counterpart are in a process of intensive development. Such process has associated quite difficult conceptual problems 5 and a fundamental and long debated one has been the question of how to define an entropy for nonequilibrium states [26]. The difficulty begins with the question of how to properly define the macrostate of the system beyond the local equilibrium regime. Partially successful attempts reside in phenomenological irreversible thermodynamics beyond the classical theory [5] T and the MaxEnt-NESOM $[14 ; 16]$. In the previous sections we have discussed this question in the framework provided by these formalisms. We have briefly described the MaxEnt-NESOM in section IITwhere we commented upon the question of the choice of the basic variables and truncation procedures $\Gamma$ which allow for contracted descriptions of the system. Irreversible evolution from an initial state of preparation [Cf. Eq. (6)] is attained through an imposed property on the Lagrange multipliers than the method introducesTin such a way that it incorporates in an ad hoc manner Prigogine's principle of dynamic condition for dissipativity [14;16]. A nonlinear generalized transport theory of large scope follows from the NESOM [Cf. Eqs. (15)] [29]. We recall that the MaxEnt-NESOM may be considered as contained in Jaynes' Predictive Statistical Physics implying in a variational method related to Information Theory [15].

The statistical foundations for phenomenological irreversible thermodynamics been described in section IIIT where the MaxEnt entropy and the MaxEnt-entropy production functions are introduced. They are expressed in terms of the chosen set of basic macrovariables deemed appropriate for the description of the nonequilibrium thermodynamic state of the system. This MaxEnt entropy is $\Gamma$ in a mechano-statistical sense $\Gamma$ a coarse-grained one. related to the logarithm of the fine-grained Gibbs' NSD. It follows from the projection of the latter onto the subspace spanned by the basic set of dynamical variables $\Gamma$ whose average values are at each instant of time the constraints imposed by the information used to characterize the macrostate of the system (See Fig. 1). In the approach of del Rio and Garcia-Colin [41] Tthe equivalent 
of this procedure is interpreted as defining the probability distribution on the microcanonical cell determined only by the constraints imposed on the system. In this sense there is no ambiguity in the procedure of coarsegraining Tprovided the NSD is determined only through such constraints.

The MaxEnt entropy is expressed as the average of the logarithm of the auxiliary Gibbsian distribution $\bar{\rho}$. This is precisely in Eq. (7) the first contribution to the total fine-grained NSDT and the one that defines the averages of the basic variables at each time $t$ [Cf. Eq. (4)]. The other term $\Gamma \rho_{w}^{\prime} \Gamma$ controls the irreversible evolution of those variables. As shownTit is this deviation of the NSD from the coarse-grained one $\bar{\rho}$ Г that determines MaxEnt - entropy production. The partial contribution $\rho_{w}^{\prime}$ contains the information on the microscopic processes governed by $H^{\prime}$ in Eq. (1) Twhereas $\bar{\rho}$ is related to macroscopic relaxation [Cf. Eq. (II.1) in Appendix II] [7]. Also in section III an irreversibility criterion consisting of a generalized $\mathcal{H}$-theorem Tnamely Eq. (37) Tis derived. Furthermore Tthe MaxEnt entropy is always largerTor at most equal Tto Gibbs entropyГas a result of the coarse-graining imposed by the use of the contracted macroscopic description of the system. More generally according to results to be reported in a future paper [43] $\Gamma$ it appears to be possible to show that there exists a hierarchy of values of entropy that increase in correspondence with a decrease in the number of elements of the basic set of variablesTnamely[the different degrees of constraints. In that way it seems to be possible to offer a possible characterization of Rosenfeld's complementarity principle of dynamical and statistical descriptions [20].

Finally $\mathrm{T}$ we have stressed that at present there is note a conclusive demonstration that the MaxEnt entropy production is instantaneously positive definite. The proof we presented in last section only ensures what we have called a weak principle of local positive entropy production $\Gamma$ which states that as the system evolves in time the local MaxEnt entropy production is predominantly positive definite.

In conclusionT the MaxEnt-NESOM in the formulation presented in section II constitutes a very powerful $\Gamma$ concise $\Gamma$ and practical mechano-statistical formu- lation which provides microscopic foundations to phenomenological nonlinear irreversible thermodynamics. The thermodynamical variables are introduced as constraints in the variational procedure (MaxEnt) for the derivation of the NSD which then depends on these variables $\Gamma$ through the Lagrange multipliers $\Gamma$ and the corresponding dynamical quantities. The MaxEnt entropy to be equated to the phenomenological one $\mathrm{Cde}-$ pends on these variables and arises from a projected part of the logarithm of the NSD $\Gamma$ which is $\ln \bar{\rho}(\Gamma \mid t, 0)$ (as shown in Fig. 1). As noticed $\Gamma$ this process is a coarse-graining procedure that restricts one to have as only accessible microstates in phase space those in the subspace spanned by the basic dynamic variables generated by the symmetry condition of Eq. (2). Irreversible effects are contained in the complementary part of the NSDTnamely $\rho_{w}^{\prime} \Gamma$ as it is demonstrated by the proven generalized $\mathcal{H}$-theorem. Furthermore $\Gamma$ the MaxEnt local entropy-production function is predominantly positive definite $\mathrm{T}$ but in any case these results can not be connected with the formulation of the second law $\Gamma$ until a clear cut definition of the entropy function in nonlinear thermodynamics for systems arbitrarily away from equilibrium is obtained. This statement has to be further clarified since here the second law must necessarily be understood as some extension of Clausius formulation which involves changes between two equilibrium states and $S$ is the calorimetric entropy which is uniquely defined. We emphasize once more that in non-equilibrium states it is very likely that many different definitions of entropy are feasible depending essentially on how to obtain - in some sense - a complete set of macrovariables that may unequivocally characterize the macrostate of the system $\Gamma$ and thus agreeing with Meixner conjecture [26]. We stress the point $\Gamma$ on which we have already commented upon $\Gamma$ that in MaxEnt-NESOM an approximate complete set of thermodynamic variables can be obtained once an appropriate criterion for the truncation procedure in the choice of these basic variables can be develop in each particular problem under consideration: as noticed an example involving highly photoexcited plasma in semiconductors is given in references [18] and [31].

We gratefully acknowledge financial support to our 
Group provided in different opportunities by the São Paulo State Research Foundation (FAPESP); the National Research Council (CNPq); the Ministry of Plan- ning (Finep); Unicamp Foundation (FAEP); and the John Simon Guggenheim Memorial Foundation (USA).

\section{Appendix I: Derivation of Eq. (27)}

Introducing in Eq. (26) the form of the equations of evolution for the basic variables as given by Eq. (16) Гit may be noticed that

$$
\begin{gathered}
\sum_{j=1}^{n} \int d^{3} r F_{j}(\vec{r}, t)\left[J_{j}^{(0)}(\vec{r}, t)+J_{j}^{(1)}(\vec{r}, t)\right]= \\
=\sum_{j=1}^{n} \int d^{3} r F_{j}(\vec{r}, t) \int d \Gamma\left\{P_{j}(\Gamma ; \vec{r}), H_{0}+H^{\prime}\right\} \bar{\rho}(\Gamma \mid t, 0) \\
=\int d \Gamma\left\{\ln \bar{\rho}(\Gamma \mid t, 0), H_{0}+H^{\prime}\right\} \bar{\rho}(\Gamma \mid t, 0) \\
=\int d \Gamma\{\bar{\rho}(\Gamma \mid t, 0), \ln \bar{\rho}(\Gamma \mid t, 0)\}\left(H_{0}+H^{\prime}\right)=0,
\end{gathered}
$$

because of the fact that the invariance under permutation used in the last two lines follows after integration by parts and taking $\bar{\rho}$ as null on the boundaries of phase space Tand finally the Poisson bracket of $\bar{\rho}$ and its logarithm is null. Therefore it follows Eq.(27).

\section{Appendix II: Alternative form of the MaxEnt-entropy production}

Using Eq. (17c) together with Eq. (18) and Eqs. (12) and (13) we can write the MaxEnt-entropy production in the form

$$
\begin{gathered}
\sigma(t)=\sum_{j=1}^{n} \int d^{3} r F_{j}(\vec{r}, t) \int d \Gamma\left\{P_{j}(\Gamma ; \vec{r}), H^{\prime}\right\} \rho_{w}^{\prime}(\Gamma \mid t)= \\
=\sum_{j=1}^{n} \int d^{3} r F_{j}(\vec{r}, t) \int_{t_{0}}^{t} d t^{\prime} \mathcal{W}\left(t, t^{\prime}\right)\left\{\left\{P_{j}(\Gamma ; \vec{r}), H^{\prime}\right\} ; \hat{\sigma}\left(\Gamma \mid t^{\prime}, t^{\prime}-t\right) \mid t\right\}= \\
=\sum_{k=1}^{\infty} \int_{t_{0}}^{t} d t^{\prime} \mathcal{W}\left(t, t^{\prime}\right)\left(\hat{\sigma}(\Gamma \mid t, 0) ; \hat{\sigma}\left(\Gamma \mid t^{\prime}, t^{\prime}-t\right) \mid t\right)^{(k)}
\end{gathered}
$$

where

$$
\begin{gathered}
\left(\hat{\sigma}(\Gamma \mid t, 0) ; \hat{\sigma}\left(\Gamma \mid t^{\prime}, t^{\prime}-t\right) \mid t\right)^{(k)}=\frac{1}{k !} \int d \Gamma \hat{\sigma}(\Gamma \mid t, 0) \hat{\sigma}\left(\Gamma \mid t^{\prime}, t^{\prime}-t\right) \\
\int_{t_{0}}^{t} d t_{1} \mathcal{W}\left(t, t_{1}\right) \hat{\sigma}\left(\Gamma \mid t_{1}, t_{1}-t\right) \ldots
\end{gathered}
$$




$$
\ldots \int_{t_{0}}^{t} d t_{k-1} \mathcal{W}\left(t, t_{k-1}\right) \hat{\sigma}\left(\Gamma \mid t_{k-1}, t_{k-1}-t\right) \bar{\rho}(\Gamma \mid t, 0)
$$

Equation (II.2) provides an expression for the MaxEnt-entropy production function that takes the form of a series of terms involving correlations of higher and higher order of the quantity of Eq. (9). This implies in the interesting fact that it is composed of higher and higher orders in the relaxation processes. MoreoverTthese expressions contain memory effects.

\section{References}

[1] E.g. R.R. Alfano, (editor), Semiconductors Probed by Ultrafast Laser Spectroscopy, Vols. 1 and 2 (Academic, New York, 1984); Biological Probed by Ultrafast Laser Spectroscopy (Academic, New York, 1982).

[2] E.g. G. Nicolis, in: The New Physics; P. Davies, Ed. (Cambridge Univ. Press, Cambridge, 1989); G. Nicolis and I. Prigogine, Exploring Complexit (Freeman, San Francisco, 1989); M.C. Cross and P.C. Hohenberg, Rev. Mod. Phys. 65, 851 (1993).

[3] E.g., S.R. de Groot and P. Mazur, Nonequilibrium Thermodynamics (North Holland, Amsterdam, 1962).

[4] C. Truesdell, Rational Thermodynamics (Mc GrawHill, New York, 1985).

[5] E.g., I. Müller and T. Ruggeri, Extended Thermodynamics (Springer, Berlin, 1993); D. Jou, J. CasasVazquez, and G. Lebon, Rep. Prog. Phys. 51, 1105 (1988); J. Non-Equil. Thermodyn. (Overview of Recent Bibliography), 17, 383 (1992); Extended Irreversible Thermodynamics (Springer, Berlin, 1993; second revised and enlarged edition 1996); L.S. Garcia-Colin and F.J. Uribe, J. Non-Equil. Thermodyn. 16, 89 (1991).

[6] R. Zwanzig, Ann. Rev. Phys. Chem. 16, 67 (1965); Kinam (Mexico) 3, 5 (1981); in: Perspectives in Statistical Physics, H.R. Ravechè, Ed. (North Holland, Amsterdam, 1981).

[7] H. Mori, I. Oppenheim, and J. Ross, in: Studies in Statistical Mechanics I, J. de Boer and G.E. Uhlenbeck, Eds. (North Holland, Amsterdam, 1962).

[8] J.A. McLennan, Phys. Fluids 4, 1319 (1961); Adv. Chem. Phys. 5, 261 (1963).

[9] S.V. Peletminskii and A.A. Yatsenko, Zh. Ekps. Teor. Fiz. 53, 1327 (1967) [Soviet Phys. - JETP 26, 773 (1968)]; also A.I. Akhiezer and S.V. Peletminskii, Methods of Statistical Physics (Pergamon, Oxford, 1981).

[10] D.N. Zubarev, Neravnovesnaia Statisticheskaia Termodinamika (Izd. Nauka, Moscow, 1971) [English Transl.: Nonequilibrium Statistical Thermodynamics Plenum - Consultants Bureau, New York, 1974], Chapter IV; D.N. Zubarev, V. Morosov and G. Röpke, Statistical Mechanics of Nonequilibrium Processes, Vols. 1 and 2 (Akademie Verlag, Berlin, 1996 and 1997).

[11] B. Robertson, Phys. Rev. 144, 151 (1966); also in: The Maximum Entropy Formalism, R. D. Levine and M. Tribus. Eds. (MIT Press, Cambridge, MA, 1978).
[12] K. Kawasaki and J.D. Gunton, Phys. Rev. A8, 2048 (1972).

[13] H. Grabert, Z. Phys. B27, 95 (1977); Projection Operator Tecniques in Nonequilibrium Statistical Thermodynamics (Springer, Berlin, 1981).

[14] R. Luzzi and A.R. Vasconcellos, Fortschr. Phys./Prog. Phys. 38, 887 (1990).

[15] E.T. Jaynes, in: Frontiers of Nonequilibrium Statistical Physics, G. T. Moore and M. O. Scully, Eds. (Plenum, New York, 1986); in: Complex Systems: Operational Approaches, H. Haken, Ed. (Springer, Berlin, 1985); in: The Maximum Entropy Formalism, R.D. Levine and M. Tribus, Eds. (MIT Press, Cambridge, MA, 1978), in: E.T. Jaynes' Papers on Probability, Statistics, and StatisticaL Physics, D. Rosenkrantz, Ed. (Reidel, Dordrecht, 1983).

[16] J.G. Ramos, A.R. Vasconcellos and R. Luzzi, Fortschr. Phys./Prog. Phys. 43, 205 (1995).

[17] A. Hobson. J. Chem. Phys. 45, 1352 (1966); Ann J. Phys. 34, 411 (1996); R.E. Nettleton, J. Phys. A 22, 5281 (1989); Physica A 158, 672 (1989); J. Chem. Phys. 93, 8247 (1990); Phys. Rev. A42, 4622 (1990); S. Af. J. Phys. 14, (1991).

[18] L.S. Garcia-Colin, A.R. Vasconcellos and R. Luzzi, J. Non-Equilib. Thermodyn. 19, 24 (1994); A.R. Vasconcellos, R. Luzzi and L.S. Garcia-Colin, Phys. Rev. A 43, 6622 (1991); ibid. 43, 6633 (1991); R. Luzzi, A.R. Vasconcellos, D. Jour and J. Casas-Vasquez, J. Chem. Phys 107, 7383 (1997).

[19] E.g. I. Prigogine, From Being to Becoming (Freeman, San Francisco, 1980); S. Hawkings, in: 1990-Yearbook of Science and the Future (Encyclopaedia Britannica, Chicago, 1989).

[20] L. Rosenfeld, in A Question of Physics, edited by P. Buckley and F.D. Peat (Univ. Toronto Press, Toronto, 1979); in Proc. Int. School of Physics "Enrico Fermi", Course XIV, edited by P. Caldirola (Academic, New York, 1960); Acta Phys. Polonica 15, 3 (1955); R. Luzzi, J.G. Ramos and A.R. Vasconcellos, Phys. Rev. E 57, 244 (1998).

[21] See for example, J. L. Lebowitz, Physics Today, 36, 32 (1983).

[22] J.P. Dougherty, in Maximum Entropy and Bayesian Methods, edited by J. Skilling (Kluwer, Dordrecht, 1989); Phil. Trans. Roy. Soc. A 346, 259 (1994); Stud. Hist. Phil. Sci. 24, 843 (1993).

[23] M.C. Mackey, Rev. Mod. Phys. 61, 981 (1989).

[24] H.H. Hasegawa and D.J. Driebe, Phys. Rev. E 50, 1781 (1994). 
[25] J.L. Lebowitz, Physics Today (Letters Section) 47, (11), 115 (1994).

[26] J. Meixner, Rheol. Acta 12, 465 (1973); also, in Foundations of Continuum Thermodynamics, edited by J.J. Delgado, M.R. Nina, and J.W. Whitelaw (McMillan, London, 1974), p. 129.

[27] H. Grad, Comm. Pure Appl. Math. 14, 323 (1961).

[28] The literature is quite broad; a recent book by M.C. Mackey, Time's Arrow: The Origin of Thermodynamic Behavior (Springer, Berlin, 1992) provides a good review. See also J.L. del Rio and L.S. Garcia-Colin, Phys. Rev. E 48, 819 (1993) and references therein.

[29] L. Lauck, A.R. Vasconcellos and R. Luzzi, Physica A168, 789 (1990).

[30] H. Mori, Prog. Theor. Phys. (Japan) 33, 423 (1965).

[31] E.g., A.C. Algarte, A.R. Vasconcellos and R. Luzzi, Phys. Stat. Sol. (b) 173, 487 (1992).

[32] R. Luzzi, A.R. Vasconcellos, J. Casas-Vazquez and D. Jou, J. Chem. Phys. 107, 7383 (1997).

[33] I. Prigogine, Acta Phys. Austriaca, Suppl. X, 401 (1973).

[34] For example, D.N. Zubarev and V.P. Kalashnikov, Physica 56, 345 (1971); V.P. Vstovskii, Teor. Mat. Fiz. 21, 376 (1974) [Theor. Math. Phys. (USSR) 21, 1214 (1975); D.N. Zubarev, Modern Methods of the Statistical Theory of Nonequilibrium Processes, Reviews of Science and Technology: Modern Problems of Mathematics, Vol. 15, pp. 131-226 (in Russian), edited by R.B. Gamkreludze (Izd. Nauka, Moscow, 1980) [English Transl.: J. Soviet Math. 16, 1509 (1981)]; see also reference [22].
[35] M.A. Tenan, A.R. Vasconcellos and R. Luzzi, Fortschr. Phys./Prog. Phys 45, 1 (1997).

[36] N.N. Bogoliubov, in: Studies in Statiscal Mechanics I, J. de Boer and G.E. Uhlenbeck, Eds. (North Holland, Amsterdam, 1962); for additional discussions and applications see: G.E. Uhlenbeck, in: Lectures in Statistical Mechanics, M. Kac, Ed. (Am. Math. Soc., Providence, RI, 1963); L.L. Buishvili and M.D. Zviadadze, Physica 59, 697 (1972); A.R. Vasconcellos, A.C. Algarte and R. Luzzi, Physica A 156, 517 (1990).

[37] D. Forster, Hydrodynamic Fluctuations, Broken Symmetry, and Correlation Functions (Benjamin, Reading, MA, 1975).

[38] D.N. Zubarev, in reference [10], see Chapter IV, Section 25.1; also V.P. Kalashnikov, Teor. Mat. Fiz. 34, 412 (1978) [Theor. Math. Phys. (USSR) 34, 263 (1978)].

[39] D.N. Zubarev, in reference [10], see Chapter IV, Section 22.4., p. 334 .

[40] R. Jancel, Foundations of Classical and Quantum Statistical Mechanics (Pergamon, Oxford, 1963).

[41] J.L. del Rio and L.S. Garcia-Colin, Phys. Rev. A 43, 6657 (1991); ibid. E 48, 819 (1993).

[42] J.G. Ramos, A.R. Vasconcellos and R. Luzzi, IFGW Internal Report (1998), and future publication.

[43] J.G. Ramos, A.R. Vasconcellos and R. Luzzi, IFGW Internal Report (1998), and future publication. 